\title{
Oltrefremente Poesia: Vittorio Stella Rilegge la Poetica di Virgillito ${ }^{I}$
}

Sergio Romanelli $^{2}$

ABSTRACT: In quest'articolo si intende analizzare criticamente la rilettura della poetica di Rina Sara Virgillito operata dal saggista italiano Vittorio Stella in occasione della pubblicazione di una antologia da lui curata di poemi della poeta intellettuale milanese.

PAROLE-CHIAVE: critica letteraria; poesia italiana contemporanea; Rina Sara Virgillito; Vittorio Stella.

1. Quest'articolo è un adattamento della conferenza presentata a Firenze nel 2005 nel corso degli Incontri di giugno su Scrittura e Memoria tenutisi all'Archivio di Stato.

2. Universidade Federal de Santa Catarina

sergio_roma70@yahoo.com 
RESUMO: Neste artigo pretende-se analisar a releitura da poética de Rina Sara Virgillito realizada pelo ensaísta italiano Vittorio Stella por ocasião da publicação de uma antologia que organizou dos poemas da poeta intelectual milanesa.

PALAVRAS-CHAVE: critica literária; poesia italiana contemporânea, Rina Sara Virgillito; Vittorio Stella.

ABSTRACT: This article will critically analyze Rina Sara Virgillito poetry re read by the Italian essayist Vittorio Stella when he organized and published a poetic anthology of that intellectual Milanese poet.

KEYWORDS: literary criticism, contemporary Italian poetry; Rina Sara Virgillito; Vittorio Stella. 
"Come tuono comprimersi nel limite/ poi con fragore romper via $[\ldots] /$ - questo sarebbe Poesia - [...]”, traduceva Rina Sara Virgillito da Emily Dickinson nei suoi ultimi mesi di vita, in un'ennesima sfida con la potenza della parola poetica; e ancora dalla stessa poesia (la 1247), “[...] o Amore - i due giungono coevi -/ entrambi li provi e nessuno-/ ne esperimenti uno e ti finisci - / perché nessuno vede Dio e vive -" (DICKINSON, 2002: p. 100).

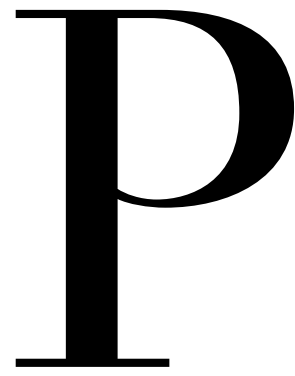

oesia e amore sarebbero, quindi, incarnazioni del divino sulla terra che il poeta, un privilegiato suo malgrado, vive e in loro si esaurisce, lasciando, però, a noi, non fortuiti, ma fortunati lettori, la forma visibile di questi sofisticati eventi. Non si perde il laccio, sentenzia Virgillito, o non dovrebbe perdersi, ma sempre più raramente Poesia e Amore giungono coevi e, se giungono, immediatamente fuggono via impercepiti, non trovano ascolto; ogni creatura si nasconde - ancora da Dickinson -, per reggere alla visione ci vuole coraggio. In un'epoca, non solo quella storica, ma anche intellettuale, sempre meno attenta alla poesia, o in genere alla belleza dell'arte, che non sceglie como canale espressivo i mezzi veloci e tecnologici della moderna comunicazione, ma quelli lenti della pagina stampata, la pubblicazione di un'antologia poetica assume, e non dovrebbe, le caratteristiche di un evento, e quindi di qualcosa di raro, insperato e inatteso. Ancor più inatteso quando si tratta della produzione di

3. Rina Sara Virgillito, Il láttice, la luce. Poesie scelte a cura e con introduzione di Vittorio Stella, Caltanissetta-Roma, Salvatore Sciascia Editore, 2003, pp. 140, euro 11,00. 
una poetessa, intellettuale e traduttrice, come Rina Sara Virgillito, apprezzata, letta e commentata da grandi critici e intellettuali, ma poco, purtroppo, dal grande pubblico.

Questa antologia, curata da un critico ed intellettuale di spessore come Vittorio Stella, è un ulteriore e importante passo verso la scoperta e la diffusione di una voce poetica di rara potenza nel panorama della letteratuta italiana del Novecento che, noi, 'amanti' e 'poeti', salutiamo con gioia, finalmente pronti a soddisfare la nostra necessità di ascolto di nuove sillabazioni. È opportuno qui ricordare come, per quel che concerne la divulgazione, molto sia già stato fatto da Ernestina Pellegrini, prima e dopo la morte di Virgillito. Si segnalano, oltre ai numerosi saggi critici su riviste e libri, soprattutto la voce sul Dizionario critico della letteratura italiana del Novecento, curato da E. Ghidetti e G. Luti (1997), e la prima monografia sulla poeta milanese, ma bergamasca d'adozione, il volume Rina Sara Virgillito. Poetica, Testi Inediti, Inventario delle carte, pubblicato nel 2001 nella prestigiosa serie Sussidi Eruditi, che, oltre a costituire un importante strumento per quanti, per studio o per curiosità, vogliano approssimarsi al mondo poetico di questa straordinaria intellettuale, raccoglie l'inventario delle carte e della biblioteca (2.697 volumi) depositati dopo la sua morte, avvenuta il 12 agosto del 1996, dall'amica ed erede testamentaria Sonia Giorgi, all'Archivio della scrittura delle donne in Toscana dal 1861, presso l'Archivio di Stato di Firenze.

Nonostante si registrino tesi di laurea e di dottorato in Italia (Greta Perletti) e in Brasile (Sergio Romanelli) e la pubblicazione e divulgazione di articoli su Virgillito in Italia e all'estero, l'attenzione dedicata a questa poeta, è decisamente ancora insufficiente. Per questo, il libro Il láttice, la luce, curato da Vittorio Stella, assume particolare importanza. Il testo si pregia di un'introduzione attenta e dettagliata sull'attività poetica di Virgillito, inserita nel più ampio ambito intellettuale e estetico della cultura italiana della seconda metà del Novecento. Il libro, nelle intenzioni del curatore, vuol essere

[...] un attendibile - e sia pur sommaria - immagine della modulazione di una personalità poetica che, in più di quarant'anni, ha vissuto non brevi periodi di silenzio (riempiti peraltro dalla notevolissima attività di traduttrice e dal corrispondente ampliamento della già vasta sfera culturale) e ne è riapparsa con una prospettiva nuova, benché sedimentata a lungo. (STELLA, 2003, pp. 14-15). 
L'antologia, oltre a riproporre poesie già pubblicate, soprattutto in La conchiglia del 1962 e in Incarnazioni del fuoco del 1991, presenta due poesie composte negli ultimi mesi di vita, Senza nome ed Eva, ed offre inoltre una scelta nutrita di poesie inedite. Questi inediti, come lo stesso curatore tiene a specificare, appartengono soprattutto ad un periodo di tempo che va dal 1948 al 1966 e molti appaiono nella versione non definitiva. I manoscritti presentano, infatti, numerose varianti a penna, con annotazioni dell'autrice a margine del testo.

Una testimonianza della genesi poetica di Virgillito è la poesia bis Nuova primavera (p. 110), composta il 9 maggio del '54, probabilmente una prima versione, che ha a fronte una seconda versione con un nuovo titolo, Primavera sulle mura (II), della primavera del 1954 e poi rivista il 28 luglio del 1956. Un piccolo documento, questo, che rivela i segni di un processo di composizione creativa complesso e rigoroso che molto può interessare a quegli studiosi della genesi della scrittura letteraria e delle peculiari strategie formali messe in atto dagli scrittori nel tentativo di concretizzare la loro straordinaria percezione della realtà.

Nella sua introduzione critica, il curatore individua due poli, allo stesso tempo opposti e complementari, nella produzione di Virgillito. Il primo ruota intorno alla raccolta La conchiglia, pubblicata nel 1962, scelta e curata da Leonardo Sciascia, che rientrerebbe nella fase che, cautamente, Stella definisce post-ermetica, ovvero una fase che contiene in sé tracce di quel fenomeno piuttosto limitato, ma i cui motivi perdureranno oltre questo breve arco di tempo, nella letteratura italiana che va dagli anni ' 30 alla Guerra e che vede in Ungaretti, Montale e Luzi i più grandi predecessori e che ha come referente privilegiato la poesia francese del secondo Ottocento e del primo Novecento. Non a caso, infatti, la poesia di Virgillito si caratterizza per le forti interconnessioni con quella, appunto, di Montale, Luzi e Sereni; ma, soprattutto, con il primo.

Si rammenta come proprio il poeta ligure costituì un modello fondamentale per Virgillito, che lo considerava una sorta di guida spirituale, nonostate ne temesse la statura intellettuale e l'abilità poetica. La loro amicizia nacque nel 1946, quando Virgillito, allora insegnante, gli inviò un piccolo saggio sulla poesia montaliana. Il saggio, che rimase nel cassetto per quarant'anni, vedrà la luce solo nel 1991 con il titolo La luce di Montale. Lo stesso poeta affermò di essersi rivelato a se tesso leggendo le parole acute 
e sensibili di Virgillito. La giovane e sconosciuta scrittrice riuscì a ribaltare prospetticamente la concezione pessimistica legata, anche fin troppo retoricamente, al tema del 'male di vivere', mediante un' acuta ed originale disamina che ruotava intorno alle figure femminili della poesia montaliana, riuscendo a mostrare il rovescio religioso e positivo della sua tessitura tematico-stilistica. Montale vinse la ritrosia di Virgillito ad esporsi e ad esporre agli altri la forma della sua eccezionale creatività, quei poemi che aveva timidamente inviato al poeta ligure e che lo stupirono e interessarono a tal punto da vincere la sua ritrosia, questa volta, a rispondere a chicchessia, fino ad invitarla presso la redazione del Corriere della Sera con il quale in quel periodo collaborava. Smosso finalmente il difficile, ma essenziale, meccanismo delle coincidenze, il progetto poetico di Virgillito prese corpo e, grazie all'interessamento di Carlo Bo, che ne fece anche la prefazione, uscì, nel 1954, la prima raccolta, I gorni del sole.

Prima della pubblicazione, nel 1962, della raccolta La conchiglia, si segnalano la versione, del 1945, di La vita della Vergine e altre poesie di Rainer Maria Rilke e, nel 1957, gli Epigrammi greci, scelti liberamente dalla Antologia Palatina. Queste due pubblicazioni sono le prime testimoninanze di un'attività di traduzione che accompagnerà quella poetica e che si intensificherà assumendo sempre più i connotati di una vera e propria esigenza di provarsi con la parola e con la potenza creativa di altri autori, e anche traduttori, per abbeverarsi all'immaginario altrui e trarne nuovi motivi, stilistici, linguistici e tematici, scoprendo nelle sillabe di altre poetiche le conferme di affinità più che elettive.

Si diceva, quindi, che La conchiglia è perno e simbolo, secondo Stella, della prima fase, quella giovanile, di Virgillito. Il lessico della raccolta pubblicata nel '62 per le edizioni Leonardo Sciascia è la natura: “[...] stagioni, paesaggi, luci, vibrazioni che ne plasmano il mutamento e bruciano nell'immagine quanto residuo deteriormente naturalistico possa insidiare la descrizione [...]" (STELLA, 2003: p. 8). Questa insistenza tematica è riscontrabile già con uno sguardo veloce ai titoli delle poesie ed ha però come sostrato appena accennato un sentimento esistenziale condizionante, l'amore che umanizza e facilita il processo di interiorizzazione dei modi e delle forme della natura, che altro non è se non la metafora del nostro animo. Per il curatore, proprio in questa raccolta si trovano i prodromi di quel 'sentimento' soggiacente alle Incarnazioni del 
fuoco, senza però possederne la stessa tensione visionaria e mistica. Si potrebbe dire, un po' liricamente, che La conchiglia è un preludio, al chiar di luna, della visionarietà crudele e oggettiva delle Incarnazioni ormai prive del conforto dell' astro notturno. Se nella Conchiglia proprio la natura con la sua fenomenologia era ciò che effondeva vita ad angoli, strade, pieghe nelle vesti delle donne, generando così sentimenti nel lettore che altro non vedeva, nelle diverse rifrazioni di luce su quei paesaggi, che la metafora del proprio animo; nelle Incarnazioni, la natura si rivela nel suo aspetto doppio di purificatrice e forza terrificante; le sue manifestazioni, in questo caso, non effondono più solamente la luce che rivela le pieghe dell'animo nelle foglie e per le strade, ma sono lo sfondo della scissione di quell'animo umano difronte alla necessità di salvezza e la percezione della propria inadeguatezza rispetto alla grandezza del divino a cui anela. La manifestazione della natura è la constatazione permanente della prezenza in tutto, negli adocchiamenti celestiali e negli inferni sottocemento, del Dio che mai completamente si rivela.

Con Incarnazioni del fuoco si arriva, secondo Stella, al secondo polo della poetica di Virgillito, quella che si definirebbe matura. È stato un lungo e ricco 'percorso di scintille' quello che ha portato l'autrice alla consolidazione e concretizzazione di quella, che dai più, e considerata la summa della sua poiesi.

Il ventennio che va dalla fine degli anni '70 ai primi anni ' 90 è un periodo molto importante e intenso in cui, e cito Stella, "L'opera di Virgillito avrà svolgimenti considerevoli, affonderà senza soste lo sguardo in rappresentazioni veementi e insieme meditative, tesaurizzerà avidamente i propri contenuti [...]" (2003, p.10). Come accadde durante tutta la sua vita letteraria, anche, e soprattutto, nell'ultimo ventennio della sua esistenza, l'attività di scrittrice e quella di traduttrice corsero parallele e a volte si intrecciarono, influenzandosi reciprocamente: nel 1976 pubblicò, infatti, Il testamento e la ballata degli impiccati di François Villon; nel 1984 la nuova raccolta di poemi Nel grembo dell'attimo; nello stesso anno la versione parziale dei Sonetti d'amore di Shakespeare, pubblicata poi in versione integrale nel 1988 e apprezzata dai più rigorosi anglisti; nel 1986 ancora letteratura inglese con le traduzioni dei Sonetti dal portoghese di E. B. Browning; nel 1991 le Incarnazioni del fuoco e nel 1994, l'ultima raccolta pubblicata in vita, L'albero di luce. Usciranno postumi le bellisme traduzioni 
dei Sonetti a Orfeo di Rilke (2001) e di 114 poesie di Emily Dickinson (2002).

La larga base culturale acquisita in quei lunghi anni e gli esercizi di traspoetizzazione da altri autori permisero a Virgillio di affinare le sue 'armi' poetiche in vista di quella che da molti è estata considerata la sua opera 'necessaria', Le incarnazini del fuoco. Di quest'ultima scrive Luzi nel risvolto di copertina:

Non c'è alcuna freccia di direzione né una mappa di orientamento sulla soglia della sua caverna mistica: in modo abrupto Sara Virgillito ci risucchia all'interno del suo poema alchemico comunicandoci immediatamente il suo ritmo. Il poema, che ha come tema il fuoco, il principio vitale e metamorfico, infatti è concepito come azione pitica e organizzato di conseguenza nel flusso metrico di un continuo esaltato e affocato trasalimento. (VIRGILLITO, 1991).

Stella, nella sua introduzione, sottolinea proprio come la lettura di Luzi e poi, e più approfonditamente, quella di Pellegrini abbiano captato la molteplicità di motivi culturali, religiosi e intellettuali presenti in questo libro. Il curatore dichiara esplicitamente essere la sua una nuova lettura della poetica virgillitiana, rispetto alle pur auterevoli, ma parziali, analisi fatte in precedenza da Montale e Bo.

Ma in che cosa consiste la novità di questa nuova raccolta di Virgillito? Per il curatore la novità sta nella "[...] maggiore e diversa ambizione, segnata da una intransigenza morale-intellettuale, da un'incessante sete di verità" (STELLA, 2003, p. 13). Esiste un nucleo ideatore di questa esperienza mistica che nasce però da una precisa ratio ispiratrice. Il luogo d'origine animatore di questa esperienza poetica sarebbe quindi, come la chiama Stella, una 'ragione generativa' di ascendenze cristiane, ma imbevuta dell'immaginario esoterico, buddhistico e sostenuta dalla psicologia analitica junghiana. Un sostrato, questo, di cui sono testimonianza i libri trovati nella biblioteca personale della poeta, dove spiccano, fra gli altri, le molteplici edizioni della Divina Commedia dantesca e i numerosissimi - se ne conteranno alla fine quasi un centinaio - testi su e di Karol Woijtyla, a conferma della larga base biblico-testamentaria che distingue le Incarnazioni. Si può quindi dire, confortati da Stella, che nel caso delle Incarnazioni ci si trovi davanti ad un itinerarium mentis in Deum permeato di reminiscenze soprat- 
tutto, e non a caso, dantesche. Un percorso nel quale si inscena il tragico dramma degli opposti, la necessaria e struggente volontà di raggiungere la congiunzione, l'unione con il principio immanente, il Dio del quale non si è mai all'altezza. Il tema dell'unione, afferma Stella, occupa l'opera intera e le dà senso. L'unione, come suggerisce il titolo, fra le 'incarnazioni' e il 'fuoco' passa irrevocabilmente attraverso il corpo che senza respingere nulla fa di tutto oggetto della sua conoscenza. La consapevolezza razionale della rivelazione inintelligibile del Dio è l'ossimoro ontologico di questa esperienza di fuoco. Sulla ineliminabile bipolarità della condizione del tutto si fonda l'avventura corporea di questo io poetico disperso nel 'nondove', in quella Illocality, parafrasando Dickinson, che è testimonianza dell'onnipresenza del divino che non sceglie luoghi precisi per svelarsi. Questa, azzardando un neologismo, 'illocazione', è espressa da Virgillito con parole nuove e toponimi che tentano disperatamente di fissare un diverso punto di riferimento, quello che solo a lei si rivela e che non riesce a definire. Per questo forse, per l'impossibilità di descrivere uno spazio incommensurabile, Virgillito usa unire in un modo inusuale e sgrammaticato parole ed avverbi per crearne di più adeguati: il 'nondove', il 'nonessere', il 'nonsisadove', l'aldisopra', il 'nonfinire'. Questa sua nuova toponomastica è forse il tentativo di concretizzare un luogo che non esiste o che supera tutti quelli esistenti. La sillaba costituirà l'asse principale di questo nuovo dis-ordine del mondo: è in lei - misura metrica del verso - che viene conservata l'energia originaria con il suo potenziale esplosivo. Per dare forma, anche graficamente, a questa decostruzione dell'ordine dell'universo, Virgillito opta sempre più frequentemente per versi, o versicoli, come preferisce definirli Stella, a volte composti solamente da singole parole, contrassegnati da una sintassi tronca, interrotta, reticente. Il tono ellittico, i troncamenti, il monolinguismo, porterebbero, secondo il curatore, ad un nuovo ermetismo di Virgillito. Per quel che riguarda il ritmo di queste composizioni, il curatore lo definisce 'forte', ad esprimere quel paradosso che è la base fondante della fede. Infatti, la necessità di essere inintelligibile alla ragione umana è il principio sufficiente della sua esistenza.

L'esperienza di questo poema lascia al lettore (quasi un sopravvissuto) la certezza che la poesia non deve (o non può) possedere nessuna verità ultima, se non il privilegio di portare in sé l'eco di quella verità. L'esperienza della lettura di questa raccolta 
e della scelta operata da Stella lascia al critico e al lettore, o dovrebbe, la consapevolezza di trovarsi davanti ad una personalità creativa qui soltanto accennata nella sua complessità e nel suo valore. L'esperienza limitata, per forza di cose, di un'antologia come questa dovrebbe lasciarci innapagati e curiosi di cercare tutto il resto di un'attività poetica vasta e fuori dal comune, che non ha trovato posto qui. L'esperienza della scoperta di una voce poetica di tal valore si fa anche e soprattutto grazie a libri come questo che hanno il coraggio di mostrare che la poesia ha forza, ha lettori, ma non ha spazio sufficiente. Alla poesia, comunque, di spazio non gliene serve molto, solo quello fisico per farsi forma, dal momento che la sua sostanza, come dice Virgillito, va oltre i limiti fisici indispensabili a realizzarla. Se la sua essenza è il 'nondove', la sua durata è l' 'oltrefremente'.

\section{Riferimenti bibliografici}

DICKINSON, E. Poesie. Tradotte da Rina Sara Virgillito. Milano: Garzanti, 2002.

EPIGRAMMI GRECI. Traduzione di Rina Sara Virgillito. Milano: Mantovani, 1957.

BROWNING, E. B. Sonetti dal portoghese. Traduzione di Rina Sara Virgillito. Firenze: Libreria delle donne, 1986.

PELLEGRINI, E. Sara Virgillito. In: GHIDETTI, E.; LUTI, G. (org.). Dizionario critico della letteratura italiana Del Novecento. Roma: Editori Riuniti, 1997, p. 915-7.

PELLEGRINI, E.; BIAGIOLI, B. Rina Sara Virgillito: poetica, testi inediti, inventario delle carte. Roma: Ed. Di Storia e Letteratura, 2001.

RILKE, R. M. La vita della Vergine e altre poesie. Traduzione di Rina Sara Virgillito. Con 20 litografie originali a colori di A. Martini. Milano: Editoriale italiana, 1945.

. M. Sonetti a Orfeo. Traduzione di Rina Sara Virgillito. Milano: Garzanti, 2000.

ROMANELLI, S. Biografia di un'estasi. Merope. Pescara, Ud'A, Anno XIII - N. 33-34 maggio-settembre 2001, p. 207-9. setembro, 2002, p. 12-3.

Rina Sara Virgillito: una mistica del Novecento. Mosaico italiano, ano I, n. 3, . Mediações poéticas: Tolentino e Virgillito. In: MEDIAÇÕES. VIII Congresso Internacional Abralic. Anais plenárias/semiplenárias/simpósios. Disponível em cd-rom.

.. Da poeta a poeta. Note a margine di una traduzione. Rina Sara Virgillito traduce Emily Dickinson. Mosaico Italiano, anno II, numero 9, ottobre 2003, p. 6-7. 
. Una quieta vulcanica vita. (L' immaginario siciliano nella poesia di Rina Sara Virgillito). In: ABPI 2003 ANAIS do X Congresso Nacional de Professores de Italiano IV Encontro Internacional de Italianística. Florianópolis, 10 a 14 de setembro de 2003. Florianópolis: UFSC, 2004, p. 303-9. Disponível em cd-rom.

. O movimento tradutório na obra de Rina Sara Virgillito. Inventário, n.3 dezembro 2004. Disponível no site: www.inventario.ufba.br

. Desvendando um labirinto: as 'traduções' de Rina Sara Virgillito. Manuscrítica. Revista de Crítica Genética. São Paulo, APML, n. 13, 2005, p. 181-93.

SHAKESPEARE, W. Sonetti d'amore. Traduzione di Rina Sara Virgillito. Roma, Newton Compton, 1984.

. I sonetti. Traduzione di Rina Sara Virgillito. Roma: G. T. E. Newton, 1988.

VILLON, F. Il Testamento e la Ballata degli impiccati. Traduzione di Rina Sara Virgillito. Milano: Rusconi, 1976.

VIRGILLITO, R. S. I giorni del sole. Presentazione di carlo Bo e un disegno di E. Montale. Urbino: Istituto d'arte, 1954.

. La conchiglia. Caltanissetta-Roma: Sciascia, 1962.

. I fiori del cardo. Milano: Scheiwiller, 1976.

. Nel grembo dell'attimo. Introduzione di Carlo Bo. Firenze: Nuove edizioni Enrico Vallecchi, 1984.

. Introduzione. In: SHAKESPEARE, W. I sonetti. Traduzione di Rina Sara Virgillito. Roma: G. T. E. Newton, 1988, p. 7-22.

. La luce di Montale. Milano, Edizioni Paoline, 1990.

. Incarnazioni del fuoco. Introduzione di Ernestina Pellegrini e una nota di Mario Luzi. Bergamo, Moretti e Vitali editori, 1991.

. L'albero di luce. Introduzione di Ernestina Pellegrini e con tre trittici di Silva Felci. Bergamo, Edizioni El Bagatt, 1994.

Il lattice, la luce. Poesie scelte. A cura e con un introduzione di Vittorio Stella.

Caltanissetta-Roma, Leonardo Sciascia Editore, 2003. 